\title{
Evidence for the activation of pyroptotic and apoptotic pathways in RPE cells associated with NLRP3 inflammasome in the rodent eye
}

\author{
Jiangyuan Gao, Jing Z. Cui, Eleanor To, Sijia Cao and Joanne A. Matsubara*
}

\begin{abstract}
Background: Age-related macular degeneration (AMD) is a devastating eye disease causing irreversible vision loss in the elderly. Retinal pigment epithelium (RPE), the primary cell type that is afflicted in AMD, undergoes programmed cell death in the late stages of the disease. However, the exact mechanisms for RPE degeneration in AMD are still unresolved. The prevailing theories consider that each cell death pathway works independently and without regulation of each other. Building upon our previous work in which we induced a short burst of inflammasome activity in vivo, we now investigate the effects of prolonged inflammasome activity on RPE cell death mechanisms in rats.

Methods: Long-Evans rats received three intravitreal injections of amyloid beta (A $\beta)$, once every 4 days, and were sacrificed at day 14. The vitreous samples were collected to assess the levels of secreted cytokines. The inflammasome activity was evaluated by both immunohistochemistry and western blot. The types of RPE cell death mechanisms were determined using specific cell death markers and morphological characterizations.

Results: We found robust inflammasome activation evident by enhanced caspase-1 immunoreactivity, augmented NFKB nuclear translocalization, increased IL-1 $\beta$ vitreal secretion, and IL-18 protein levels. Moreover, we observed elevated proteolytic cleavage of caspase-3 and gasdermin D, markers for apoptosis and pyroptosis, respectively, in RPE-choroid tissues. There was also a significant reduction in the anti-apoptotic factor, X-linked inhibitor of apoptosis protein, consistent with the overall changes of RPE cells. Morphological analysis showed phenotypic characteristics of pyroptosis including RPE cell swelling.

Conclusions: Our data suggest that two cell death pathways, pyroptosis and apoptosis, were activated in RPE cells after exposure to prolonged inflammasome activation, induced by a drusen component, $A \beta$. The involvement of two distinct cell death pathways in RPE sheds light on the potential interplay between these pathways and provides insights on the future development of therapeutic strategies for AMD.
\end{abstract}

Keywords: Age-related macular degeneration, Retinal pigment epithelium, NLRP3 inflammasome, Cell death, Amyloid beta

\footnotetext{
*Correspondence: jms@mail.ubc.ca

Department of Ophthalmology and Visual Sciences, Eye Care Centre, Faculty

of Medicine, University of British Columbia, 2550 Willow Street, Vancouver,

BC V5Z 3N9, Canada
} 


\section{Background}

Age-related macular degeneration (AMD) is a neurodegenerative disease that strikes the macula, causing irreversible blindness to people over the age of 50 in industrialized countries. The global prevalence of AMD has created substantial economic and social burden with a projected estimate of 196 million people living with AMD in 2020 [1]. Clearly, better eye care strategies need to be designed to provide health care services to those in need. Clinically, AMD can be predicted by a severity scale that is a function of drusen deposition and pigment abnormalities [2]. The accumulation of drusen both in size and in number has become a hallmark of AMD progression. As it progresses, AMD transitions from early benign stages into advanced vision-threatening stages, presenting with either choroidal neovascularization (CNV, wet form) and/or geographic atrophy (GA, dry form). Although $\mathrm{CNV}$ is the severe subtype of the advanced AMD, it is clinically managed using the antivascular endothelial growth factor (VEGF) therapy [3]. However, there is no effective treatment to slow down the more prevalent dry form, which makes up approximately $90 \%$ of all AMD cases. Retinal pigment epithelium (RPE) cell death and secondary photoreceptor degeneration are two signature changes that lead to central vision loss in GA, the advanced stage of dry AMD. Hence, it is paramount to understand the fundamental mechanisms underlying these devastating impacts to the retina, especially the ones that undermine RPE health.

Despite the lack of consensus on the exact cell death pathway(s) involved, there have been three candidate cell death mechanisms proposed to underlie RPE atrophy in GA, including necrosis, apoptosis, and pyroptosis [4-6]. Necrosis is a classic form of RPE cell death in GA, which was reported in earlier clinical-pathological studies by Sarks et al. [4], and also in basic research projects with ultrastructural and histochemical data [7]. Apoptotic RPE cell death, on the other hand, has gained substantial support from the literature in recent years. Using postmortem human eyes, Kaneko and colleagues identified the activation of caspase-3 in the RPE layer of GA eyes, but not in normal control eyes [5]. Moreover, Dunaief et al. demonstrated statistically significant differences in the number of terminal deoxynucleotidyl transferase dUTP nick end-labeling (TUNEL) positive retinal cells in postmortem retinas with AMD, compared to normal controls [8]. The third proposed mechanism of RPE death is pyroptosis, which is an inflammatory form of programmed cell death [9]. The cornerstone of pyroptosis is the activation of an intracellular multi-protein complex named the inflammasome. NLR family pyrin domain containing 3 (NLRP3) inflammasome is the most widely studied machinery and consists of NLRP3, active caspase-1, and a bridging adaptor, apoptosisassociated speck-like protein containing a carboxyterminal CARD (ASC). For the NLRP3 inflammasome to function, it requires sequential treatment of two types of pathological stimuli, (1) a priming signal to activate nuclear factor kappa B (NF-kB) and upregulate the transcription of NLRP3 and interleukin (IL)-1 $\beta$ precursor protein; (2) an activation signal to trigger the inflammasome assembly for the production of two pro-inflammatory cytokines, IL-1 $\beta$ and IL-18. The relationship between NLRP3 inflammasome and AMD pathology has been an attractive subject in the field, and current knowledge on this subject is reviewed elsewhere in detail [10]. Although all three cell death pathways described above seems to govern RPE cell fate in GA to some extent, it is still unclear whether these mechanisms act independently or in synergy.

Earlier work, including ours, demonstrated that components found in drusen, (e.g., amyloid beta, complement cascade products) increase in the aged retina [11, 12]. Previously, we have established a rat intraocular injection model to mimic the increasing amyloid beta $(A \beta)$ load associated with drusen in human eyes [13]. We demonstrated that drusen component, $A \beta$, triggers a short lasting pro-inflammatory response in RPE via the activation of NF-кB and NLRP3 inflammasome [13, 14], which can be specifically abolished by vinpocetine (an NF- $\mathrm{KB}$ inhibitor) [15]. Intriguingly, RPE cell loss is not a feature of the short lasting pro-inflammation associated with the acute $A \beta$ intravitreal model [13]. In the present study, we extended the duration of outer retina proinflammation by making sequential $A \beta$ injections, in order to better model the chronic pro-inflammatory events and associated cell death underlying the pathogenesis of the dry form of AMD.

\section{Methods \\ Preparation of oligomeric $A \beta$}

The lyophilized, synthetic $A \beta 1-40$ peptide (hereafter referred to as "A $\beta$ ") in the $\mathrm{HCl}$ salt form was purchased from American Peptide (Sunnyvale, CA). We chose A $\beta 1-40$ peptide over its structurally similar but more toxic, Alzheimer's disease (AD)-specific, form of A $\beta 1-42$ peptide based on earlier studies that demonstrated the presence of $A \beta 1-40$ in drusen deposits in postmortem human eyes [13]. Oligomeric $A \beta$ was prepared according to published protocols $[16,17]$. Briefly, the synthetic $A \beta$ peptide was first reconstituted in 1,1,1,3,3,3-hexafluoro2-propanol (HFIP, Sigma Aldrich, St. Louis, MO) and was evaporated by speed vacuum, resulting in thin transparent $A \beta$ peptide film. The $A \beta$ peptide film was then reconstituted in 100\% dimethyl sulfoxide (DMSO, Sigma Aldrich) to a concentration of $1 \mathrm{mM}$, and further diluted in pre-warmed phosphate buffered saline (PBS, pH 7.4) 
to produce the $\mathrm{A} \beta$ injection solution of $323 \mu \mathrm{M}$, equivalent to $1.4 \mu \mathrm{g} / \mu \mathrm{L}$. The injection solution was subsequently incubated at $37{ }^{\circ} \mathrm{C}$ for $48 \mathrm{~h}$ to form the oligomeric $A \beta$. Confirmation of successful oligomeric $A \beta$ formation was achieved by western blot (WB) using our published protocols [11]. Reverse $A \beta 40-1$ peptide (hereafter, referred to as "control") served as a sequence control for $A \beta$ and was prepared in the same fashion. The absence of protein bands recognized by a mouse monoclonal anti-A $\beta 1-16$ antibody (clone 6E10, Table 1) in the control solution indicated proper preparation (Additional file 1: Figure S1).

\section{Animals}

Adult female Long-Evans rats at the age of 4.5 month (Charles River, Wilmington, MA) were randomly divided into two groups. Group $1(N=16)$ comprised rats receiving intravitreal injections of $\mathrm{A} \beta(5 \mu \mathrm{L}$ at $1.4 \mu \mathrm{g} / \mu \mathrm{L}$ as previously published [13]) once every 4 days for a total of three injections. Group $2(N=16)$ rats received intravitreal injections of the control solution (reverse $A \beta 40-1$ peptide) the same way as described for group 1 . All rats were sacrificed on the 14th day after initial injection (day 14). Eyes were immediately enucleated and frozen for WB, polymerase chain reaction (PCR), and enzymelinked immunosorbent assay (ELISA) or fixed in $4 \%$ paraformaldehyde diluted in Dulbecco's PBS (Invitrogen, Carlsbad, CA) for 48-72 h prior to paraffin embedding.

\section{Immunohistochemistry (caspase-1, IL-18, NF-KB, active caspase-3)}

Paraffin-embedded rat eye tissues were processed following established protocols [13]. Sections from both the $A \beta$ and the control groups were processed simultaneously in an effort to minimize variability in immunoreactivity conditions ( $N=3$ per group). Primary antibodies recognizing total caspase-1, IL-18, NF- $\mathrm{kB}$, and active caspase-3 are described in Table 1. Non-specific isotype IgGs (Sigma Aldrich) matching the species of primary antibodies are used on negative control tissue sections. For visualization, the slides were developed using the Vector AEC substrate kit (Vector Laboratories, Burlington ON, Canada) and were counterstained with Mayer's Hematoxylin (Sigma Aldrich) for the nuclei. Sections processed simultaneously were analyzed and scored so as to avoid difference in immunostaining due to conditions such as temperature and stock of antibodies. Caspase-1, IL-18, and active caspase-3 immunoreactivity was scored in a masked fashion and semi-quantitatively based on a $0-3$ point scale. A score of 0 indicates no detectable staining above the background level as compared to the negative control sections, whereas a score of 1,2 , or 3 suggests weak, intermediate, and robust intensity of the immunoreactivity, respectively. The immunoreactivity scores of caspase-1, IL-18, and active caspase- 3 were averaged and normalized to the control group.

To detect NF- $\mathrm{kB}$ translocalization, an antibody recognizing the phosphorylated Ser 276 locus on NF-kB p65 subunit was used (Table 1). Immunoreactivity was measured quantitatively, in a masked fashion, using a $\times 60$ objective lens and $\times 10$ eyepieces. Positive RPE nuclei were identified as containing both the red AEC chromogen and blue hematoxylin counterstain, thus resulting in a purple appearance distinct from the unlabeled RPE nuclei that were blue in color due to the hematoxylin counterstain alone. The number of NF- $\mathrm{kB}$ positive nuclei was converted to percentage of all RPE nuclei in the sample area and was normalized to the control group.

\section{Suspension array for vitreal cytokines}

An ELISA-based cytokine assay for vitreal cytokines was carried out (Bio-Plex 200 System, Bio-Rad Laboratories, Hercules, CA). The assay targeted the following

Table 1 List of primary antibodies

\begin{tabular}{|c|c|c|c|c|}
\hline Antigen & Antibody & Dilution & Source & Applications \\
\hline $\begin{array}{l}\text { Amyloid-beta amino acid } \\
1-16\left(A \beta_{1-16}\right)\end{array}$ & $\begin{array}{l}\text { Mouse monoclonal } \\
\text { anti-A } \beta_{1-16} \text { (clone 6E10) }\end{array}$ & $\begin{array}{l}1: 2000 \\
1: 500\end{array}$ & BioLegend, Dedham, MA & $\begin{array}{l}\text { Western blot } \\
\text { Immunohistochemistry }\end{array}$ \\
\hline Caspase-1 & Rabbit monoclonal & $1: 300$ & Abcam, Cambridge, UK & Immunohistochemistry \\
\hline $\begin{array}{l}\text { Phosphorylated NF-KB p65 } \\
\text { (Ser 276) }\end{array}$ & Rabbit polyclonal & $1: 75$ & $\begin{array}{l}\text { Santa Cruz Biotechnology, } \\
\text { Dallas, TX }\end{array}$ & Immunohistochemistry \\
\hline Interleukin-18 (IL-18) & Rabbit polyclonal & $\begin{array}{l}1: 100 \\
1: 1000\end{array}$ & $\begin{array}{l}\text { Santa Cruz Biotechnology, } \\
\text { Dallas, TX }\end{array}$ & $\begin{array}{l}\text { Immunohistochemistry } \\
\text { Western blot }\end{array}$ \\
\hline Active caspase-3 (aCasp-3) & Rabbit monoclonal & $1: 1000$ & $\begin{array}{l}\text { Cell Signaling Technology, } \\
\text { Beverly, MA }\end{array}$ & Immunohistochemistry \\
\hline $\begin{array}{l}\text { X-linked inhibitor of apoptosis } \\
\text { (XIAP) }\end{array}$ & Mouse monoclonal & $1: 1000$ & $\begin{array}{l}\text { BD Transduction Laboratories, } \\
\text { San Jose, CA }\end{array}$ & Western blot \\
\hline Gasdermin D (GSDMD) & Mouse monoclonal & $1: 100$ & $\begin{array}{l}\text { Santa Cruz Biotechnology, } \\
\text { Dallas, TX }\end{array}$ & Western blot \\
\hline GAPDH & Mouse monoclonal & $1: 10,000$ & EMD Millipore, Billerica, MA & Western blot \\
\hline
\end{tabular}


cytokines: erythropoietin (EPO), granulocyte colony stimulating factor (G-CSF), granulocyte macrophage colony stimulating factor (GM-CSF), chemokine (C-X-C motif) ligand $1(\mathrm{GRO} / \mathrm{KC})$, interferon-gamma (IFN- $\gamma$ ), IL-1 $\alpha$, IL-1 $\beta$, IL-2, IL-4, IL-5, IL-6, IL-7, IL-10, IL-12p70, IL-13, IL-17, IL-18, macrophage colony stimulating factor (M-CSF), macrophage inflammatory protein 1alpha (MIP-1 $\alpha$ ), MIP-3 $\alpha$, regulated on activation, normal T cell expressed and secreted (RANTES), TNF- $\alpha$, and vascular endothelial growth factor (VEGF). Vitreous from rat eyes in the same group (either $A \beta$ or control) were pooled $(N=7)$. Experiments were carried out following methods in our earlier publication [15].

\section{Western blot}

To determine the level of X-linked inhibitor of apoptosis (XIAP), whole retina tissues (including neuroretina, RPE, Bruch's membrane, and choroid) from each of the two injection groups were used $(N=5)$. To investigate IL-18 secretion and gasdermin D (GSDMD) cleavage, RPE-choroid tissues from each of the two injection groups were dissected out and pooled to use $(N \geq 3)$. Tissues were homogenized in ice-cold RIPA buffer (Thermo Fisher Scientific, Waltham, MA) containing protease inhibitor cocktail (Roche Diagnostics, Indianapolis, IN). Protein lysates were run under reducing conditions and established blotting procedures were followed. Detailed information on the primary antibodies used in western blotting can be found in Table 1 [11]. As an internal protein loading control, GAPDH was detected either on the stripped membrane or using freshly thawed protein lysates (Table 1). The protein band intensity of XIAP (57 kDa), IL-18 (18 kDa), pro-GSDMD (53 kDa), N-GSDMD (30 kDa), and GAPDH (36 kDa) was individually measured using Image $(\mathrm{NIH}$, Bethesda, MD) and was converted into ratios relative to GAPDH. The final relative intensity of XIAP, IL-18, pro-GSDMD, or N-GSDMD was normalized to the control group.

\section{Reverse transcription PCR (RT-PCR)}

Total RNA of RPE-choroid was isolated from pooled rat eye tissues $(N \geq 3)$ using ultRNA Column Purification kit (Applied Biological Materials, Richmond BC, Canada). 200 ng total RNA from each injection group was reverse transcribed into cDNA using the High-Capacity cDNA Reverse Transcription kit (Applied Biosystems, Carlsbad, CA). RT-PCR was carried out on the 7500 Fast Real-time PCR System (Applied Biosystems) using the following cycling conditions: $95{ }^{\circ} \mathrm{C}$ for $30 \mathrm{~s}, 50{ }^{\circ} \mathrm{C}$ for $30 \mathrm{~s}, 72{ }^{\circ} \mathrm{C}$ for $30 \mathrm{~s}, 40$ cycles. RT-PCR primer sequences can be found in Table 2. Melting curve analysis was automatically performed right after the cycles' completion. The results were expressed as mRNA fold-change
Table $\mathbf{2}$ List of primer sequences

\begin{tabular}{lll}
\hline Gene & $\begin{array}{l}\text { Forward primer } \\
\left(5^{\prime}-3^{\prime}\right)\end{array}$ & $\begin{array}{l}\text { Reverse Primer } \\
\left(5^{\prime}-3^{\prime}\right)\end{array}$ \\
\hline $\begin{array}{l}\text { X-linked inhibitor of } \\
\text { apoptosis (XIAP) }\end{array}$ & CACACAGTCTA & CTACAACCTGT \\
Glyceraldehyde 3-phosphate & CATCTCCT & CCAGTTCT \\
dehydrogenase (GAPDH) & CTCTTGTGACAA & CCATTTGATGTT \\
\hline
\end{tabular}

relative to the control group after normalization to the reference gene, GAPDH, using the $2^{-\Delta \Delta C T}$ method.

\section{RPE morphological assessment}

To evaluate the morphological changes of RPE cells due to induced inflammasome activity, we developed a custom Photoshop algorithm to measure the area of a set length of RPE monolayer based on RPE pigmentation. For each animal group, a total of nine sections from each animal were used for the analysis. All RPE micrographs were taken under $\times 60$ magnification and subsequently cropped into $12 \mathrm{~cm} \times 2 \mathrm{~cm}$ rectangular areas for further processing. Next, choroidal pigments were manually removed and the RPE-only areas were selected by applying "fuzziness". Then, the pixel count for the cropped area was obtained. The RPE area measurement was expressed as number of pixels. This area measure was equivalent to, but more accurate than measuring the thickness of RPE monolayer.

RPE cell nuclei count and retinal thickness measurement

Following our established protocol, retinal cross sections within $200 \mu \mathrm{m}$ distance from the optic disc were chosen for the analysis because of their uniform retinal thickness regardless of embedding orientation [13, 18]. Briefly, RPE nuclei were counted by scanning the whole retinal section under $\times 20$ magnification in $10^{3} \mu \mathrm{m}$ increments. Retinal thickness was measured from the inner limiting membrane to the photoreceptor outer segments/RPE junction. The mean values of RPE nuclei count per increment, retinal thickness as well as ONL thickness were averaged over a minimum of 4-6 retinal sections per animal at each time point.

\section{Statistical analyses}

Data are presented as mean $\pm \mathrm{SD}$. Non-parametric tests were used throughout the study except the vitreal cytokine levels and RT-PCR were analyzed by one-tailed Student's $t$ test. For the non-parametric comparisons between the two groups (A $\beta$ vs control), one-tailed Mann-Whitney $U$ tests were used. All analyses were conducted with GraphPad Prism version 6 (GraphPad Software, La Jolla, CA). Statistical significance was set at $p \leq 0.05$. 


\section{Results}

Pro-inflammatory cytokine secretion in vitreous

To understand the overall inflammatory status in the rat eyes, we collected and examined the vitreous samples using an ELISA-based cytokine profile assay. We found monocytes chemoattractant protein 1 (MCP-1), chemokine (C-X-C motif) ligand 1 (GRO/KC), vascular endothelial growth factor (VEGF), and macrophage inflammatory protein 3 alpha (MIP-3 $\alpha$ ) were increased more than $50 \%$ in the $A \beta$ injected eyes compared to those in the reverse $A \beta$ injected (hereafter, referred to as "control") eyes (Fig. 1). A smaller, but significant, increase was associated with vitreal IL-1 $\beta$, a mature secreted product following NLRP3 inflammasome activation. However, IL-18, another NLRP3 inflammasome product, was downregulated in the vitreous sample from the $A \beta$ injection group compared to the control (A $\beta$ $1142.67 \pm 64.15 \mathrm{pg} / \mathrm{mL}$; control $1258.13 \pm 56.49 \mathrm{pg} / \mathrm{mL})$, which led us to further look at the inflammasome activity in $A \beta$-injected rat eyes. Measurements of the additional cytokines studied here are given in Additional file 2: Figure S2.

NLRP3 inflammasome activation by multiple $A \beta$ injections NLRP3 inflammasome activation is achieved by a sequential stimulation of a priming signal and an activation signal. To examine inflammasome activation in $\mathrm{RPE}$, we first looked at the nuclear translocalization of $\mathrm{NF}-\mathrm{kB}$, a signature event of NF- $\mathrm{BB}$ activation. Using an antibody specifically targeting the phosphorylated p65 subunit of NF- $\mathrm{BB}$, we found strong immunoreactivity in the nuclei of RPE cells in $A \beta$-injected eyes compared to control eyes (Fig. 2a-c). Next, we immunolabeled caspase-1 in the RPE layer of both $A \beta$-injected and control eyes, revealing that caspase- 1 immunoreactivity

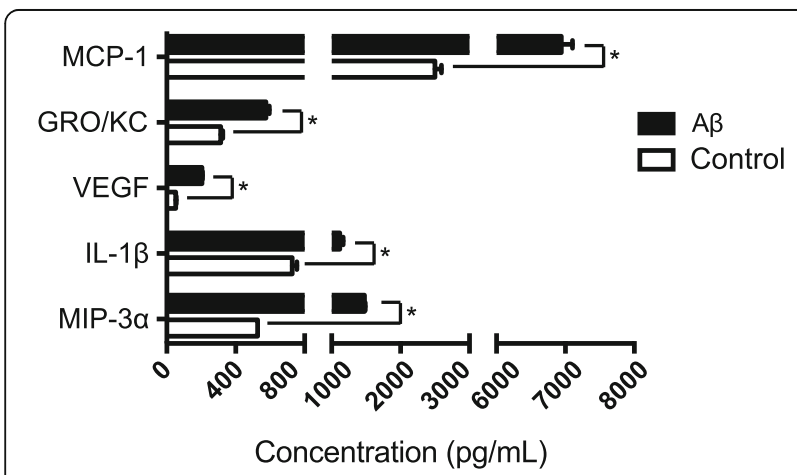

Fig. 1 Vitreal cytokine secretion following sequential intraocular injections. An ELISA-based rat cytokine assay was used to measure the vitreal cytokine levels. Cytokines with significant increase in the $A \beta$-treated eyes compared to the controls were graphed. Sequential injections of $A \beta$ led to a robust increase in chemokines (MCP-1, $\mathrm{MIP}-3 \mathrm{a}, \mathrm{GRO} / \mathrm{KC})$, inflammasome product $(\mathrm{IL}-1 \beta)$, and growth factor (VEGF). $N=7$, Student's $t$ test, ${ }^{*} p<0.05$

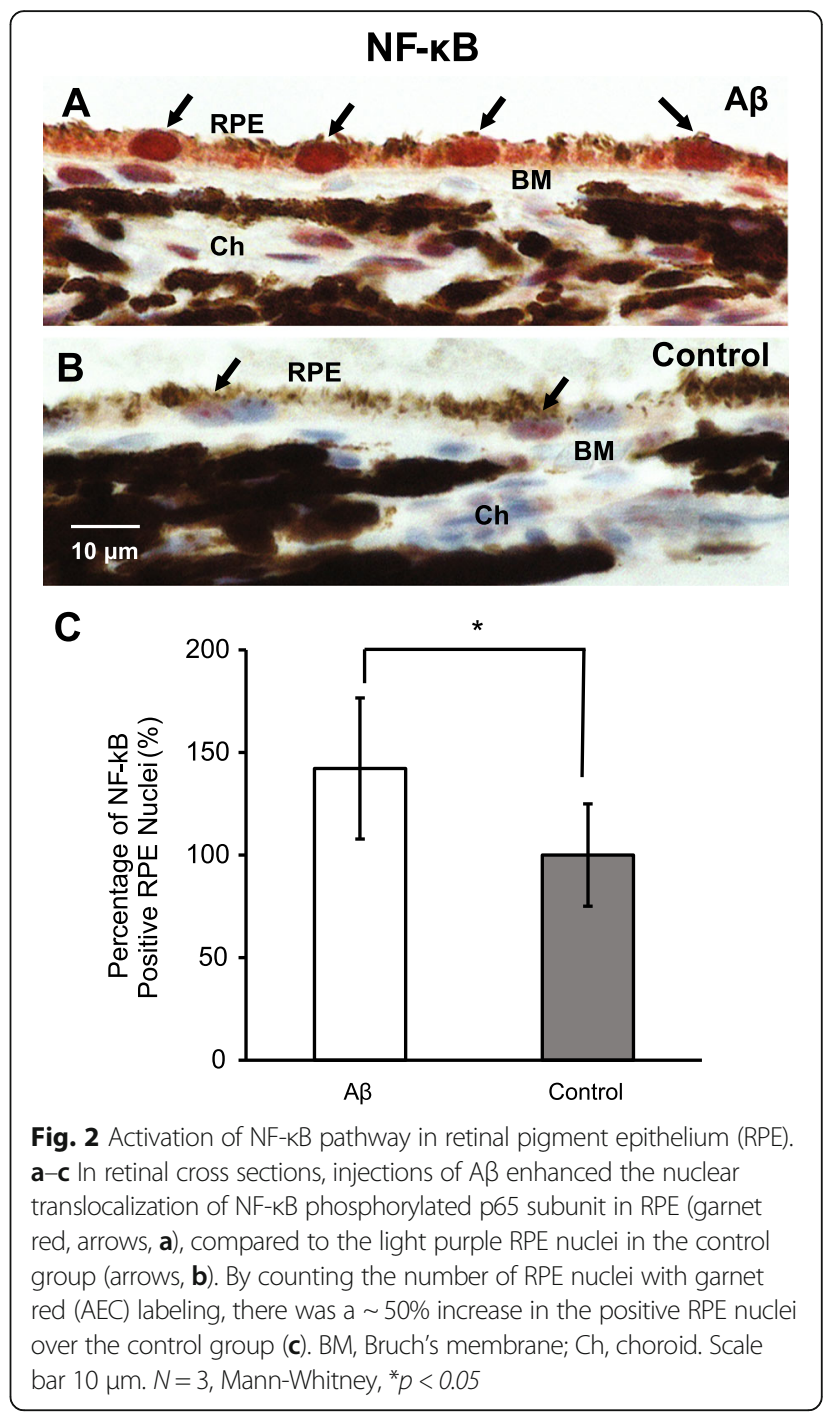

was enhanced by $77 \%$ compared to the control eyes (Fig. 3a). Consistent with the elevation of IL-1 $\beta$ in the vitreous, we found the other inflammasome activation product, IL-18, was also upregulated, showing more than 6-fold higher immunoreactivity in the RPE layer of A $\beta$-injected eyes (Fig. $3 \mathrm{~b}-\mathrm{c}$ ) and a $58 \%$ increase of IL-18 band intensity in protein lysates from the $A \beta$ injected eyes, compared to the control eyes (Fig. 3d-e).

\section{Cell death pathways triggered by $A \beta$ injections}

To study the forms of RPE cell death often seen in late AMD, especially GA, we next analyzed morphological changes in RPE after $A \beta$ injections. Using a custom Photoshop algorithm, we first applied a pigment threshold to identify the area occupied by RPE within a standard $12 \mathrm{~cm} \times 2 \mathrm{~cm}$ boxed area centered on the RPE monolayer. Next, the number of pixels within the pigment threshold of that area was obtained as an index of the area measurement occupied by the RPE. Higher 

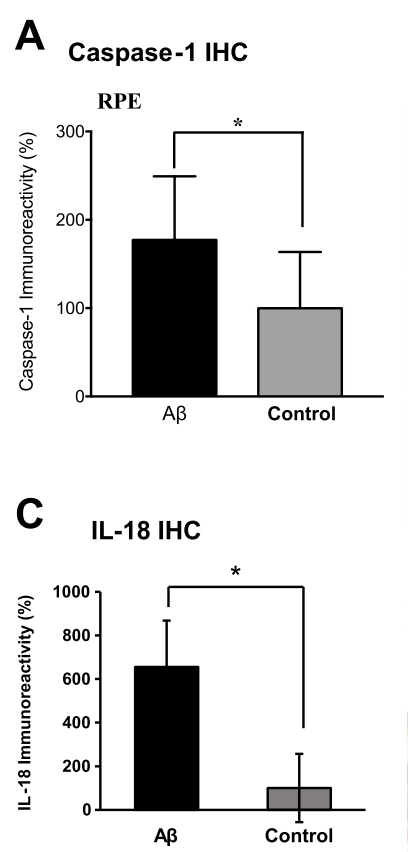

D

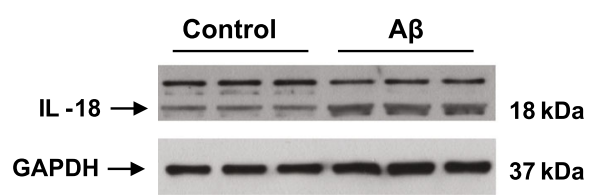

IL - 18 IHC

B $A B \quad$ Control
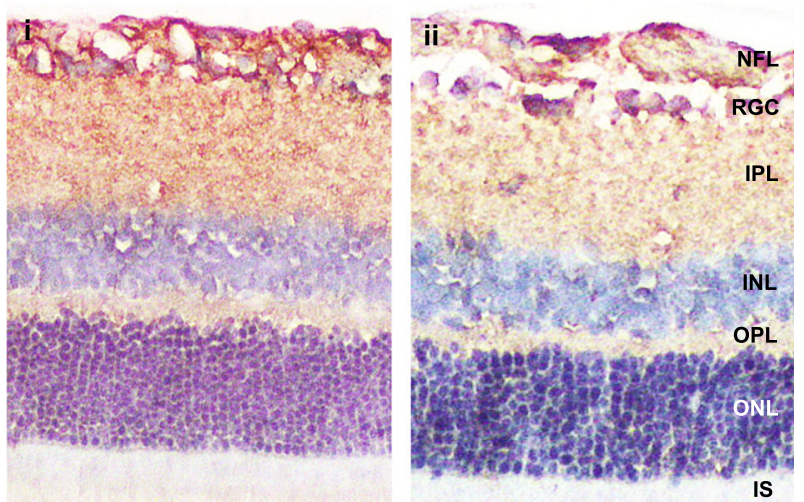

os
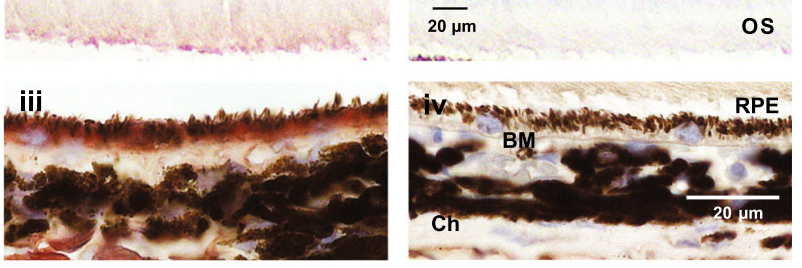

E

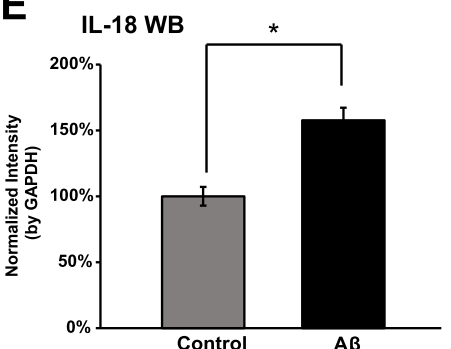

Fig. 3 Sequential AB injections promote NLRP3 inflammasome activation. a Immunoreactivity of total caspase-1 showed a 77\% increase in RPE of the $A \beta$-injected eyes compared to the control group. $\mathbf{b}-\mathbf{c}$ The Aß-injected animals had a 6-fold higher IL-18 immunoreactivity than the control group (c). Representative micrographs exhibit an overall more intense IL-18 labeling (AEC, red, panel i and ii), particularly in the RPE layer (iii and iv) (b). NFL, nerve fiber layer; RGC, retinal ganglion cells; IPL, inner plexiform layer; INL, inner nuclear layer; OPL, outer plexiform layer; ONL, outer nuclear layer; IS, inner segments; OS, outer segments; RPE, retinal pigment epithelium; BM, Bruch's membrane; Ch, choroid. Scale bars $20 \mu$ m. (d-e) IL-18 western blotting showed a $58 \%$ increase of band intensity $(\mathrm{MW}=18 \mathrm{kDa}$ ) in $\mathrm{A} \beta$ injected animals compared to the controls. Bands shown are technical triplicates of the same-pooled sample. $N \geq 3$, Mann-Whitney, ${ }^{*} p<0.05$

pixel values indicated thicker RPE monolayers. By comparing the $A \beta$-injected to the control groups, we observed a 2-fold increase in the number of pixels, suggesting a significant area increase in the RPE, presumably due to swelling of the RPE cells (Fig. 4).

To understand what caused the RPE to enlarge or swell, we prepared RPE-choroid tissue homogenates from both injection groups and tested them for gasdermin D (GSDMD), a protein executing pyroptosis and whose proteolytic cleavage is triggered by both canonical and non-canonical inflammasome activation in immune cells [19-21]. The cleaved GSDMD N-terminal fragment (N-GSDMD) at $30 \mathrm{kDa}$ was increased, while the uncleaved pro-GSDMD full-length protein at $53 \mathrm{kDa}$ was decreased, in the $\mathrm{A} \beta$ group compared to the control group (Fig. 5).
Next, we also tested for apoptosis involvement using caspase- 3 activation as a surrogate marker. By immunohistochemistry, we found 2.5- to 4.5-fold higher immunoreactivity levels of active caspase- 3 in photoreceptor inner segments and RPE of the $A \beta$ group, respectively, compared to the control group (Fig. 6a-b). Two examples of active caspase- 3 immunoreactivity from both $\mathrm{A} \beta$-injected and control eyes were provided to demonstrate the enhanced red AEC labeling in photoreceptor inner segments and RPE from A $\beta$-injected eyes (Fig. 6c). To further support this finding, we measured the mRNA and protein levels of X-chromosome-linked inhibitor of apoptosis (XIAP), a classic anti-apoptosis factor. At the transcriptional level, there was a 10-fold reduction in the $\mathrm{A} \beta$ group compared to the controls (Fig. 7a). 


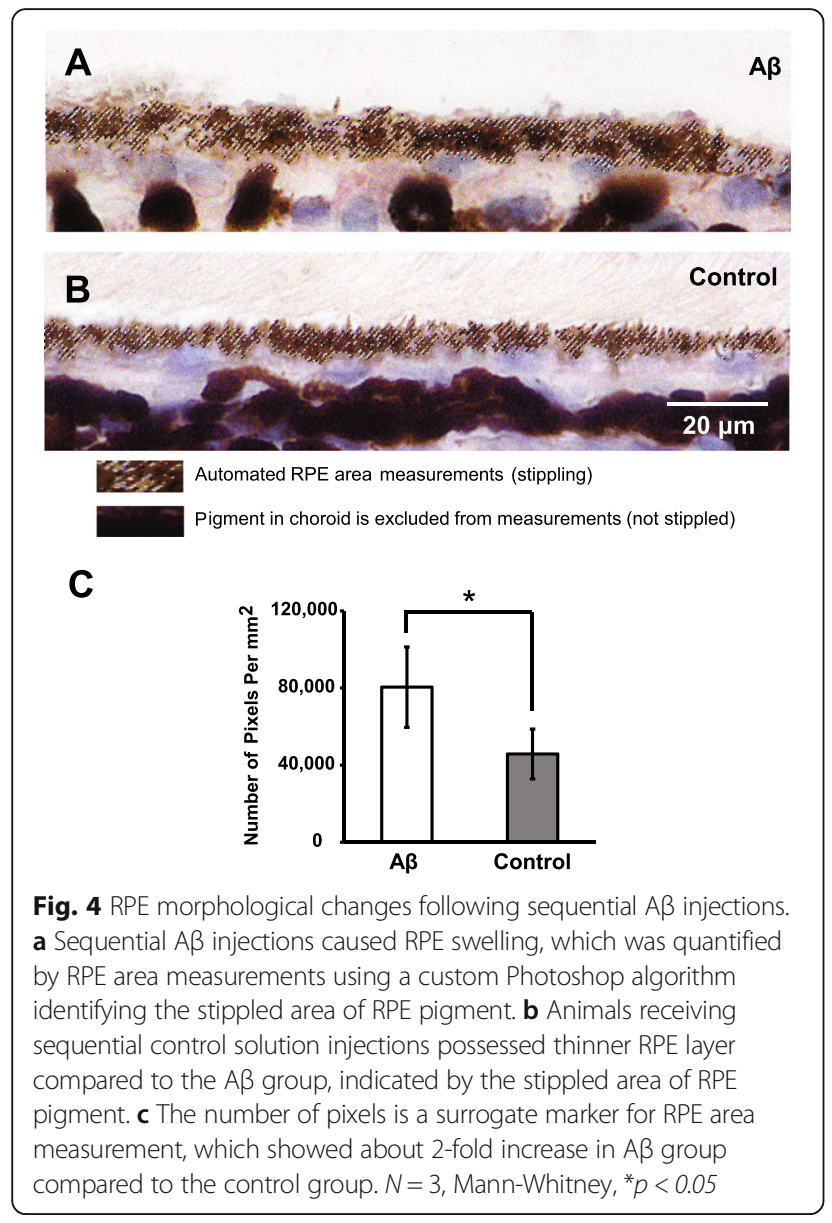

Concomitant with the mRNA data, there was a $33 \%$ reduction in XIAP protein compared to the control group (Fig. $7 \mathrm{~b}-\mathrm{c}$ ).

Finally, to determine whether the activation of pyroptotic and apoptotic pathways caused overt anatomical changes to the retina, we counted the RPE nuclei and assessed retinal thickness. Our results showed that there was no significant RPE nuclei loss or retinal thickness changes, including the ONL thickness, in the retinal cross sections of $\mathrm{A} \beta$-injected eyes compared to the control eyes (Fig. 8). These measurements indicated no overt anatomical/histological changes were evident at the time point studied here.

\section{Discussion}

In late stage AMD, RPE cells die, yet the cell death pathways responsible remain a mystery. Based on pathological specimens of AMD donor eyes, atrophic RPE cells were thought to die from a necrotic cell death pathway, in which hypopigmented RPE cells filled with membrane-bound melanolipofuscin were eliminated, resulting in the increased pigmentation and cell body enlargement in adjacent RPE cells [4]. More recently, reports from Kaneko et al. [5] and Tarallo et al. [6] highlight other cell death mechanisms (such as apoptosis and pyroptosis) that also likely contribute to atrophic RPE demise. However, the question remains as to what factors initiate the cell death cascades in AMD. In this study, using an animal model in which we mimicked pro-inflammation due to prolonged (14 days) exposure
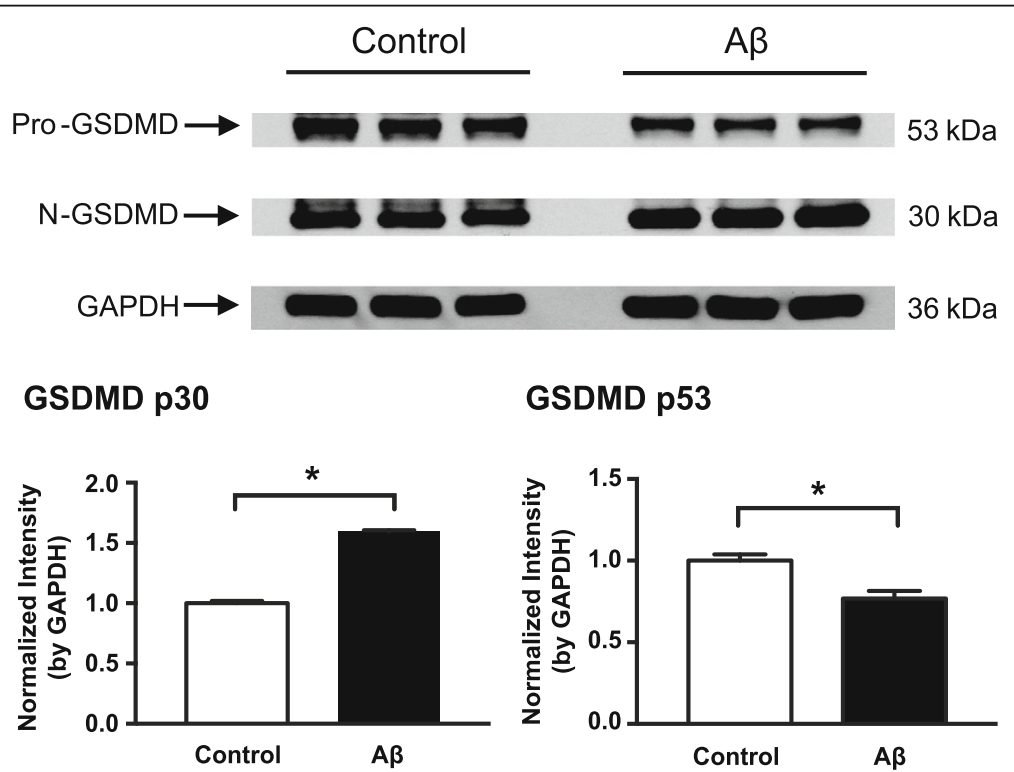

Fig. 5 Activation of GSDMD mediated pyroptosis in response to $A \beta$ stimulation. RPE-choroid protein lysates from animals receiving either $A \beta$ or control injections were probed with a GSDMD antibody. Compared to the control samples, A significantly promoted the proteolytic cleavage of pro-GSDMD (53 kDa) into N-terminal GSDMD fragment (N-GSDMD, $30 \mathrm{kDa}$ ), a signature event indicating the activation of the pyroptotic pathway. GAPDH served as a loading control (36 kDa). Bands shown are technical triplicates of the same-pooled sample. $N \geq 3$, Mann-Whitney, ${ }^{*} p<0.05$ 

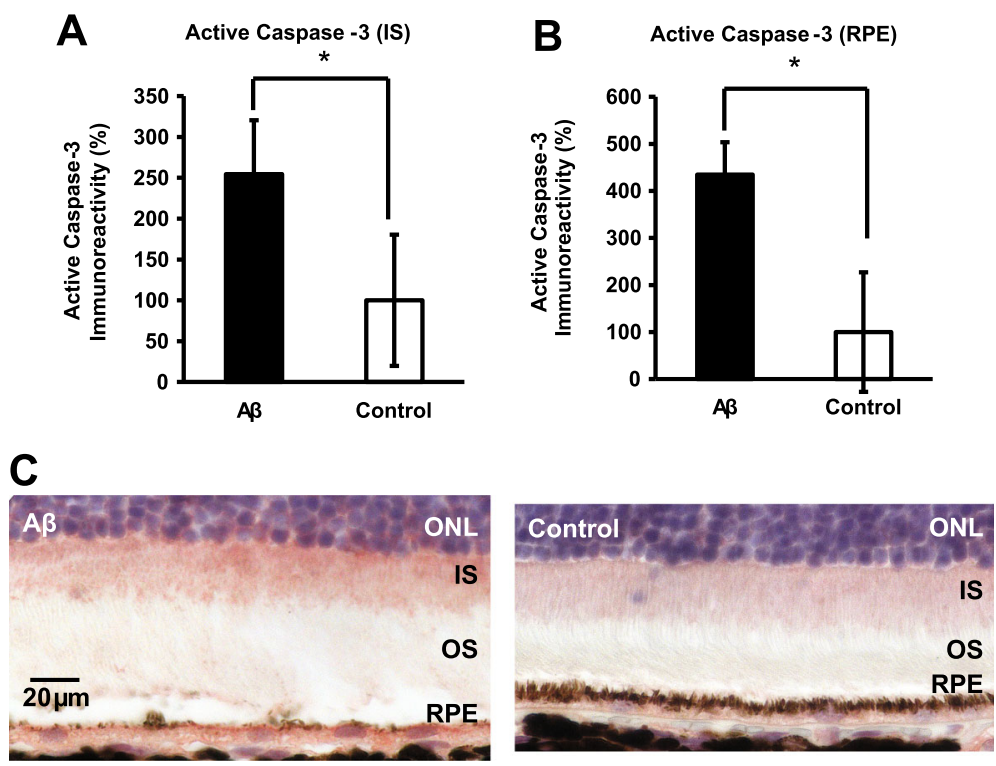

Fig. 6 Caspase-3 activation in the outer retina of A -stimulated eyes. $\mathbf{a}-\mathbf{b}$ Immunoreactivity of active/cleaved caspase-3 was 2.5- and 4.5-fold higher in the AB-injected eyes for photoreceptors' inner segments (IS) and RPE, respectively, compared to the control eyes. $N=3, \mathrm{Mann}-$ Whitney, ${ }^{*} p<0.05$. c Representative micrographs illustrated examples of the semi-quantitative grading of active caspase-3's immunoreactivity in both A $\beta$-injected and control animals. Robust caspase-3 immunoreactivity in the IS was seen in the Aß-injected retina, whereas weak and intermediate caspase-3 immunoreactivity was seen in the control eyes. ONL, outer nuclear layer; OS, outer segments. Scale bar $20 \mu \mathrm{m}$

to a drusen component, $A \beta$, we assessed the level of involvement of pyroptotic and apoptotic pathways.

\section{RPE inflammasome activation is a feature of the model}

NLRP3 inflammasome activation, one of the fundamental innate immune defense mechanisms, has recently been studied for its role in the development of AMD [22]. Our earlier study indicated that a single $A \beta$ injection resulted in a peak in pro-inflammation at 4 days post injection, but then dramatically subsided [13]. In the current study, we extended the duration of proinflammation from 4 to 14 days by making sequential injections of $A \beta$ every 4 days to achieve better retinal penetration and mimic a chronic inflammatory microenvironment in the outer retina (Additional file 3: Figure S3). Our results demonstrated that longer exposure to pro-inflammation triggered robust NF- $\mathrm{kB}$ p65 subunit translocalization in RPE nuclei, elevated levels of total caspase-1 immunoreactivity, and enhanced secretion of IL- $1 \beta$ in vitreous and increased IL-18 presence in retina. Collectively, these results support inflammasome activity in the RPE (Fig. 3, Additional file 3: Figure S3). When compared with other inflammasome studies on RPE cells including ours, the current study demonstrated significant increase of IL-18 in neuroretina and RPE-choroid, but not in the vitreous where IL- $1 \beta$ was found upregulated by more than $50 \%$ (Figs. 1 and 3, Additional file 2: Figure S2). This secretion pattern is unique in that it diversifies the downstream biological events of these inflammasome cytokines: in our previous acute model of single $A \beta$ injection, vitreal IL- 18 increased by more than 3 -fold at day 14 whereas vitreal IL- $1 \beta$ elevated by less than 50\% compared to the controls [13]. Such discrepancy in IL-1 $\beta / 18$ vitreal levels can be partially explained by the "distinct licensing requirements" for processing these two cytokines by NLRP3 inflammasomes, which might involve further regulation of caspase-11 and/or reactive oxygen species (ROS), as evident in immune cells [23]. This mechanism is also very likely to be true in RPE cells since $A \beta$ has recently been shown to induce NLRP3 inflammasome activation via NADPH oxidaseand mitochondria-dependent ROS pathway in vitro [24]. Other studies suggest that a high level of retinal IL-18 (including RPE-choroid) is more related to the cell death events in GA $[6,25]$, which support our findings that higher IL-18 immunoreactivity levels in $A \beta$-injected retina are correlated with the activation of pyroptotic pathway.

Sequential injections of $A \beta$ also provide a good model to study A $\beta$ 's role in inflammasome activation in the eye. Considered as one of the pathological hallmarks in $\mathrm{AD}$, the deposition of $A \beta$ in the $A D$ brain is associated with elevated NLRP3 inflammasome activity, particularly the elevation of IL-1 $\beta$ production [26-28]. Using microglia culture models, Halle et al. demonstrated the importance of NLRP3 inflammasome activation for the recruitment of microglia to $A \beta$ deposits in the $A D$ brain [29]. Heneka et al. further discovered that in the absence of 


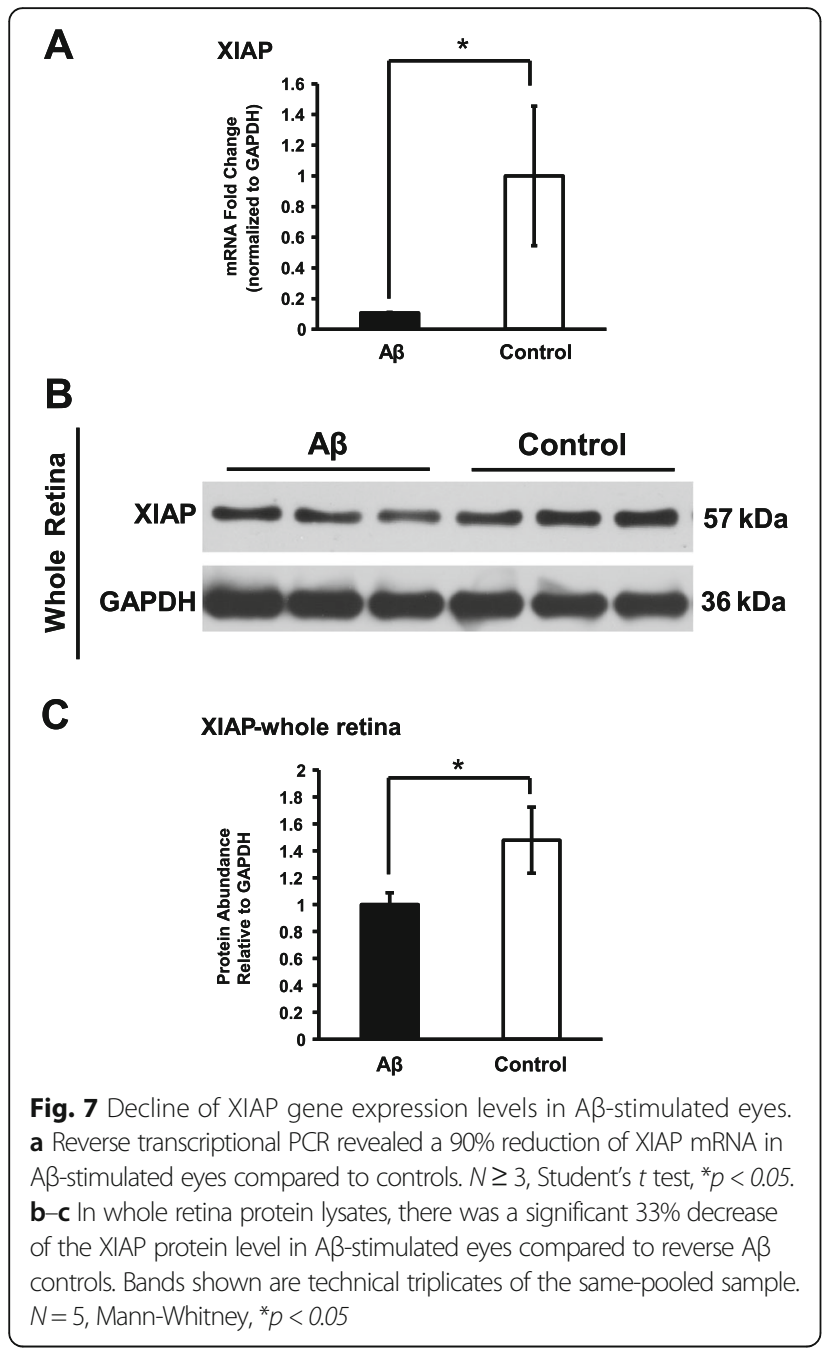

NLRP3 or caspase-1, mice carrying mutations associated with familial $\mathrm{AD}$ were largely protected from spatial memory loss, and demonstrated reduced IL- $1 \beta$ secretion and enhanced $A \beta$ clearance [27]. However, the involvement of these pathways has not been well established in the eye tissue. As a continuation from our previous studies [13], our current $A \beta$ multi-injection model recapitulates seminal features associated with each step of the NLRP3 inflammasome activation cascades. Therefore, by giving the animals sequential $A \beta$ injections, we were able to sustain $A \beta$-induced pro-inflammatory responses in the neuroretina and RPE at a comparably high level until day 14, when the presence of $A \beta$ was much diminished in the single $A \beta$ injection model [13].

\section{Pyroptosis and apoptosis may contribute to RPE cell death in this model}

The involvement of NLRP3 inflammasome activation in RPE has been studied in many different AMD models [30-33]. However, once the inflammasome is activated, little is known of the exact biological events that occur and whether these events lead to cell death. Using rodent and non-human primate models, Doyle and colleagues demonstrate the efficacy of IL-18 treatment as a potential alternative, adjuvant therapy for $\mathrm{CNV}$ [34-36]. On the other side, Ambati and collaborators showed evidence that IL-18 drives RPE degeneration after NLRP3 inflammasome activation in murine models $[6,37]$. In the current study, we also assessed the changes after activation of the NLRP3 inflammasome by $A \beta$. Intriguingly, after prolonged pro-inflammation in outer retina, we found enlarged or swollen RPE cells and significant increases in the proteolytic cleavage of fulllength GSDMD in the RPE-choroid tissues. As reported by earlier studies, the cytolytic effects of pyroptosis are mediated by the oligomerization of the GSDMD's $\mathrm{N}$-terminal fragments (N-GSDMD) in cellular membrane, resulting in the cell-burst pore formation [38]. Hence, it confirms the activation of pyroptotic pathway in the A $\beta$-injected animals [19]. Therefore, we have demonstrated the co-existence of two events that occur after inflammasome activation: the secretion of mature pro-inflammatory cytokines, including the inflammasome products (IL-18 and IL-1 $\beta$ ) and morphological and
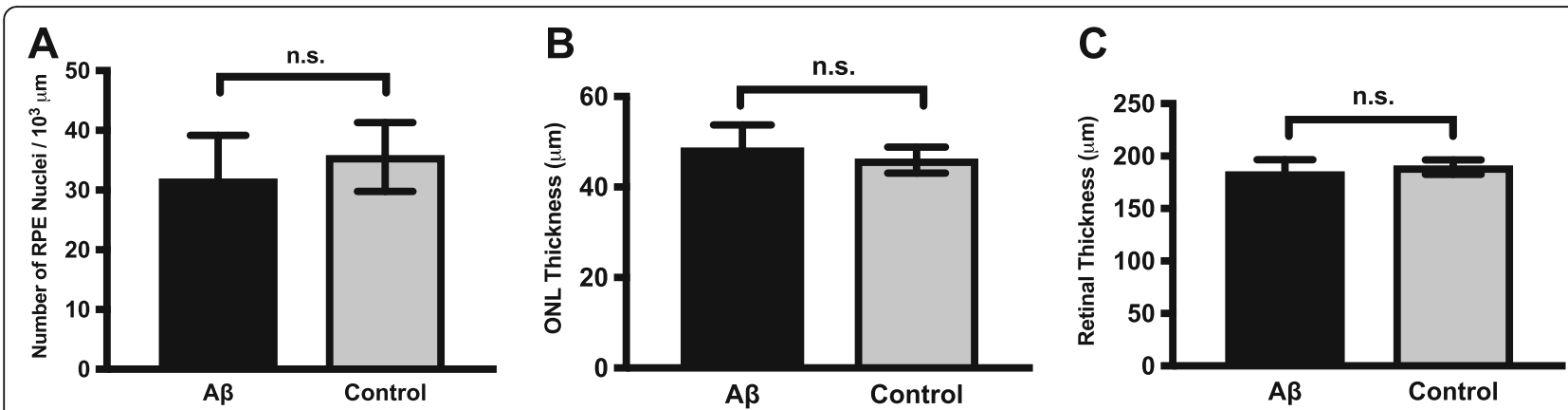

Fig. 8 No changes in RPE nuclei number and retinal thickness in A $\beta$-stimulated eyes. The number of RPE nuclei per $10^{3} \mu m$ (a), ONL thickness (b), and retinal thickness $(\mathbf{c})$ were measured in both the Aß-stimulated and control animals' eyes. No significant difference was found between the two groups in all three analytical categories ( $N=3$, Mann-Whitney) 
western blot evidence that supports the GSDMDmediated pyroptotic pathway activation in RPE cells. Such an orchestrated response has also been seen in non-ocular cell types [39].

Unlike pyroptosis, which rapidly lyses the cell, apoptosis is considered as a non-inflammatory and non-cytolytic form of programmed cell death. From a canonical point of view, pyroptosis is biochemically characterized as caspase-1 dependent and caspase-3 independent, whereas apoptosis is often caspase- 3 dependent and caspase- 1 independent. Interestingly, in the current study, we observed parallel cleavage of both caspase- 1 and caspase3 in the RPE tissue, challenging the dogma of their mutual exclusiveness. Although it is biologically impossible for one single cell to undergo both distinctive cell death pathways, it is still likely for one type of tissue, such as the RPE monolayer in the retina, to accommodate these pathways in a spatially discrete and stimulus dose-dependent manner. One study using murine bone marrow-derived macrophages exploited the potential of crosstalk between pyroptosis and apoptosis. The authors exhibited a DNA-dose dependent integral model for cell death, with apoptosis seen at a lower-dose of DNA stimulation and pyroptosis at higher doses. They further concluded that such an explicit response is regulated through caspase- 8 activation [40].

Despite the fact that our data supported the activation of both pyroptotic and apoptotic pathways in the RPEchoroid tissue of multiple $A \beta$-injected eyes, there were no significant anatomical changes as indicated by RPE nuclei counts and retinal thickness measurements (Fig. 8). All the biochemical markers used in this study (IL-18, IL-1 $\beta$, NF-kB, caspase-1, caspase-3, GSDMD, XIAP) proved changes leading towards RPE atrophy via both cell death pathways. Combined with the anatomical data, our results suggest a transition between early and middle-to-late stage of dry AMD, where RPE cells begin to show early biochemical signs of cell death and before any observable retinal structural changes.

\section{RPE-specific vs choroidal macrophage-mediated responses in vivo}

The ability to dissect RPE-choroid from neuroretina was demonstrated in our earlier work [11, 13] and those of others [41]. However, it is possible that in the RPE-choroid sample, the choroidal component with macrophages, may also contribute to the inflammasome activity in our in vivo work. Testing for activated macrophages by immunohistochemistry in paraffin sections or by microdissection of choroidal tissue alone [42] may help us better understand the migration of immune cells from choroid in our $A \beta$-injected animal groups. It is possible that the choroidal macrophages, important immune cells associated with AMD [43, 44] in combination with RPE may both work to exacerbate the chronic inflammatory milieu in the AMD eye. Therefore, future in vitro work on cultured RPE cells and choroidal macrophages will allow us to define the role of each cell type without the confounding effects from the other.

\section{Conclusions}

In conclusion, we have demonstrated the activation of two distinct cell death pathways in RPE following prolonged pro-inflammation induced by drusen component, $A \beta$. For the first time, we show that GSDMD cleavage is associated with inflammasome activation in RPE, providing the molecular basis for pyroptosis participation in this model. Consistent with other studies using postmortem human AMD donor eye tissues [6], our model recapitulates the key events in the NLRP3 inflammasome cascade. Furthermore, the presence of swollen, enlarged RPE cells in this model represents another prominent feature of RPE morphological changes during AMD progression [10, 45]. Even though the retinal morphological analysis found no evidence of ultimate RPE cell loss or retinal thinning in this model, the biochemical events revealed here point towards a critical transitional stage that may further lead to the occurrence of RPE cell death. Understanding the detailed molecular mechanisms associated with this stage will benefit future endeavors in the search of therapeutic agents to slow down, or even prevent, RPE cell death in AMD.

\section{Additional file}

Additional file 1: Figure S1. Preparation of oligomeric $A \beta$. (A) Various incubation conditions were tested for the optimal oligomeric $A \beta$ generation. Small oligomeric (dimeric and trimeric) and monomeric $A \beta$ species were generated after a shorter period of incubation time $(24 \mathrm{~h}, 100 \mu \mathrm{M})$. Note that the higher molecular $A \beta$ species began to increase after $48 \mathrm{~h}(\mathrm{MW}>$ $170 \mathrm{kDa}$ ). (B) Western blot of the $48 \mathrm{~h}$-incubated oligomeric $A \beta$ solution, prepared for intraocular injections $(7 \mu \mathrm{g} / 5 \mu \mathrm{L})$. The control peptide was prepared the same way as $A \beta$, but was non-reactive to the $6 \mathrm{E} 10$ antibody detection. (EPS $5439 \mathrm{~kb}$ )

Additional file 2: Figure S2. Additional vitreal cytokine levels following sequential $A \beta$ injections. $A$ list of rat cytokines in addition to Fig. 1 was examined in vitreous samples from both the $A \beta$-stimulated and the control animals' eyes using the ELISA-based premade assay. Unlike Fig. 1's data, all these cytokines showed less than $50 \%$ change of concentration levels between the two animal groups, many of which had even lower measurements in the A $\beta$-stimulated group, for instance, IL-18. $N=7$, Student's $t$ test, ${ }^{*} p<0.05$. RANTES, regulated on activation normal $T$ cell expressed and secreted; GM-CSF, granulocyte macrophage colony stimulating factor; EPO, erythropoietin; G-CSF, granulocyte colony stimulating factor; M-CSF, macrophage colony stimulating factor. (EPS $170 \mathrm{~kb}$ )

Additional file 3: Figure S3. Retinal distribution of injected $A \beta$. To ensure the effectiveness of sequential $A \beta$ injections, retinal cross-sections from day 14 (indicated as " $3 X$ A $\beta$ day 14 ") were stained for the injected $A \beta$ using the $6 E 10$ antibody (Table 1). As visualized by the red $A E C, A \beta$ exhibited good penetration of all retinal layers from retinal ganglion cell layer to RPE. Compared to its counterpart from our previous acute $A \beta$ model (indicated as " $1 X A \beta$ day $14^{\prime \prime}$ ), the current $3 X A \beta$ day 14 retinal cross-sections showed much stronger $A \beta$ immunoreactivity, suggesting a higher retinal $A \beta$ concentration maintained by the sequential $A \beta$ 
injections. Such robust $A B$ immunoreactivity in the $3 X A \beta$ day 14 tissue (neuroretina + RPE) was comparable to those of $1 X A \beta$ retinal tissues at days 1 and 4, with even more enhanced labeling in the outer nuclei layer. RGC, retinal ganglion cell; IPL, inner plexiform layer; INL, inner nuclei layer; OPL, outer plexiform layer; ONL, outer nuclei layer; IS/OS, inner/ outer segments. Scale bar $20 \mu \mathrm{m}$. (EPS $13727 \mathrm{~kb}$ )

\section{Abbreviations}

AD: Alzheimer's disease; AEC: 3-amino-9-ethylcarbazole; AMD: Age-related macular degeneration; ASC: Apoptosis-associated speck-like protein containing a carboxy-terminal CARD; AB: Amyloid beta; CNV: Choroidal neovascularization; DMSO: Dimethyl sulfoxide; ELISA: Enzyme-linked immunosorbent assay; GA: Geographic atrophy; GRO/KC: Chemokine (C-X-C motif) ligand 1; GSDMD: Gasdermin D; HFIP: 1,1,1,3,3,3-hexafluoro-2-propanol; IL-18: Interleukin-18; IL-1 $\beta$ : Interleukin-1 beta; MCP-1: Monocytes chemoattractant protein 1; MIP-3a: Macrophage inflammatory protein 3 alpha; NF-kB: Nuclear factor kappa B; N-GSDMD: GSDMD N-terminal fragment; NLRP3: NLR family pyrin domain containing 3; PBS: Phosphate buffered saline; PCR: Polymerase chain reaction;

RIPA: Radioimmunoprecipitation assay; RPE: Retinal pigment epithelium; TUNEL: Terminal deoxynucleotidyl transferase dUTP nick end-labeling; VEGF: Vascular endothelial growth factor; WB: Western blot; XIAP: Xchromosome linked inhibitor of apoptosis

\section{Acknowledgements}

The authors acknowledge the technical assistance provided by Alison Fong, Elliott To, Cyrus Thomas, Aikun Wang, and Matthew Wong.

\section{Funding}

The study was funded by a Canadian Institutes of Health Research operating grant to J.A.M. (MOP126195). The funding body did not participate in the design of the study, collection, analysis, and interpretation of data, and writing the manuscript in any forms.

\section{Availability of data and materials}

All data generated or analyzed during this study are included in this published article.

\section{Authors' contributions}

$J G$ performed the experiments, analyzed and interpreted the data, and wrote the manuscript. JZC designed the study, helped with the experiments and data analysis/interpretation, and contributed to manuscript revision. ET assisted with data collection, analysis, and interpretation, and edited the manuscript. SC assisted with data collection and interpretation, provided support for statistical analysis, and edited the manuscript. JAM conceived and designed the study, obtained funding, analyzed and interpreted the data, and critically revised the manuscript. All authors read and approved the final manuscript.

\section{Ethics approval}

All animal procedures were approved by the Animal Care Committee of the University of British Columbia, conformed to the guidelines of the Canadian Council on Animal Care and in accordance with the Resolution on the Use of Animals in Research of the Association of Research in Vision and Ophthalmology. Efforts were made to ensure animal welfare and to minimize their pain, distress, and discomfort whenever possible.

\section{Competing interests}

The authors declare that they have no competing interests.

\section{Publisher's Note}

Springer Nature remains neutral with regard to jurisdictional claims in published maps and institutional affiliations.

\section{Received: 24 August 2017 Accepted: 9 January 2018}

Published online: 12 January 2018

\section{References}

1. Wong WL, Su X, Li X, Cheung CM, Klein R, Cheng CY, Wong TY. Global prevalence of age-related macular degeneration and disease burden projection for 2020 and 2040: a systematic review and meta-analysis. Lancet Glob Health. 2014;2:e106-16.

2. Age-Related Eye Disease Study Research G. A simplified severity scale for age-related macular degeneration: AREDS report no. 18. Arch Ophthalmol. 2005;123:1570-4.

3. Amoaku WM, Chakravarthy U, Gale R, Gavin M, Ghanchi F, Gibson J, Harding S, Johnston RL, Kelly SP, Lotery A, et al. Defining response to anti-VEGF therapies in neovascular AMD. Eye (Lond). 2015;29:721-31.

4. Sarks JP, Sarks SH, Killingsworth MC. Evolution of geographic atrophy of the retinal pigment epithelium. Eye (Lond). 1988;2(Pt 5):552-77.

5. Kaneko H, Dridi S, Tarallo V, Gelfand BD, Fowler BJ, Cho WG, Kleinman ME, Ponicsan SL, Hauswirth WW, Chiodo VA, et al. DICER1 deficit induces Alu RNA toxicity in age-related macular degeneration. Nature. 2011;471:325-30.

6. Tarallo V, Hirano Y, Gelfand BD, Dridi S, Kerur N, Kim Y, Cho WG, Kaneko H, Fowler BJ, Bogdanovich S, et al. DICER1 loss and Alu RNA induce agerelated macular degeneration via the NLRP3 inflammasome and MyD88. Cell. 2012;149:847-59.

7. Hageman GS, Luthert PJ, Victor Chong NH, Johnson LV, Anderson DH, Mullins RF. An integrated hypothesis that considers drusen as biomarkers of immunemediated processes at the RPE-Bruch's membrane interface in aging and age-related macular degeneration. Prog Retin Eye Res. 2001;20:705-32.

8. Dunaief $J$, Dentchev T, Ying GS, Milam AH. The role of apoptosis in age-related macular degeneration. Arch Ophthalmol. 2002;120:1435-42.

9. Bergsbaken T, Fink SL, Cookson BT. Pyroptosis: host cell death and inflammation. Nat Rev Microbiol. 2009;7:99-109.

10. Gao J, Liu RT, Cao S, Cui JZ, Wang A, To E, Matsubara JA. NLRP3 inflammasome: activation and regulation in age-related macular degeneration. Mediat Inflamm. 2015;2015:690243.

11. Zhao T, Gao J, Van J, To E, Wang A, Cao S, Cui JZ, Guo JP, Lee M, McGeer $\mathrm{PL}$, Matsubara JA. Age-related increases in amyloid beta and membrane attack complex: evidence of inflammasome activation in the rodent eye. J Neuroinflammation. 2015:12:121.

12. Hoh Kam J, Lenassi E, Jeffery G. Viewing ageing eyes: diverse sites of amyloid Beta accumulation in the ageing mouse retina and the upregulation of macrophages. PLoS One. 2010;5(10):e13127.

13. Liu RT, Gao J, Cao S, Sandhu N, Cui JZ, Chou CL, Fang E, Matsubara JA. Inflammatory mediators induced by amyloid-beta in the retina and RPE in vivo: implications for inflammasome activation in age-related macular degeneration. Invest Ophthalmol Vis Sci. 2013;54:2225-37.

14. Kurji KH, Cui JZ, Lin T, Harriman D, Prasad SS, Kojic L, Matsubara JA. Microarray analysis identifies changes in inflammatory gene expression in response to amyloid-beta stimulation of cultured human retinal pigment epithelial cells. Invest Ophthalmol Vis Sci. 2010;51:1151-63.

15. Liu RT, Wang A, To E, Gao J, Cao S, Cui JZ, Matsubara JA. Vinpocetine inhibits amyloid-beta induced activation of NF-kappaB, NLRP3 inflammasome and cytokine production in retinal pigment epithelial cells. Exp Eye Res. 2014;127:49-58.

16. Sarroukh R, Cerf E, Derclaye S, Dufrene YF, Goormaghtigh E, Ruysschaert JM, Raussens $V$. Transformation of amyloid beta(1-40) oligomers into fibrils is characterized by a major change in secondary structure. Cell Mol Life Sci. 2011;68:1429-38.

17. Stine WB, Jungbauer $L, Y u C$, LaDu MJ. Preparing synthetic Abeta in different aggregation states. Methods Mol Biol. 2011;670:13-32.

18. Guo L, Normando EM, Nizari S, Lara D, Cordeiro MF. Tracking longitudinal retinal changes in experimental ocular hypertension using the CSLO and spectral domain-OCT. Invest Ophthalmol Vis Sci. 2010;51:6504-13.

19. Shi J, Zhao Y, Wang K, Shi X, Wang Y, Huang H, Zhuang Y, Cai T, Wang F, Shao F. Cleavage of GSDMD by inflammatory caspases determines pyroptotic cell death. Nature. 2015;526:660-5.

20. He WT, Wan H, Hu L, Chen P, Wang X, Huang Z, Yang ZH, Zhong CQ, Han J. Gasdermin $D$ is an executor of pyroptosis and required for interleukin-1beta secretion. Cell Res. 2015;25:1285-98.

21. Kayagaki N, Stowe IB, Lee BL, O'Rourke K, Anderson K, Warming S, Cuellar T, Haley B, Roose-Girma M, Phung QT, et al. Caspase-11 cleaves gasdermin D for non-canonical inflammasome signalling. Nature. 2015;526:666-71.

22. Celkova L, Doyle SL, Campbell M. NLRP3 inflammasome and pathobiology in AMD. J Clin Med. 2015;4:172-92.

23. Schmidt RL, Lenz LL. Distinct licensing of IL-18 and IL-1beta secretion in response to NLRP3 inflammasome activation. PLoS One. 2012;7:e45186.

24. Wang K, Yao Y, Zhu X, Zhang K, Zhou F, Zhu L. Amyloid $\beta$ induces NLRP3 inflammasome activation in retinal pigment epithelial cells via 
NADPH oxidase- and mitochondria-dependent ROS production. J Biochem Mol Toxicol. 2016;31:e21887.

25. Kim Y, Tarallo V, Kerur N, Yasuma T, Gelfand BD, Bastos-Carvalho A, Hirano Y, Yasuma R, Mizutani T, Fowler BJ, et al. DICER1/Alu RNA dysmetabolism induces caspase-8-mediated cell death in age-related macular degeneration. Proc Natl Acad Sci U S A. 2014;111:16082-7.

26. Olsen I, Singhrao SK. Inflammasome involvement in Alzheimer's disease. J Alzheimers Dis. 2016;54:45-53.

27. Heneka MT, Kummer MP, Stutz A, Delekate A, Schwartz S, Vieira-Saecker A, Griep A, Axt D, Remus A, Tzeng TC, et al. NLRP3 is activated in Alzheimer's disease and contributes to pathology in APP/PS1 mice. Nature. 2013;493:674-8.

28. Salminen A, Ojala J, Suuronen T, Kaarniranta K, Kauppinen A. Amyloid-beta oligomers set fire to inflammasomes and induce Alzheimer's pathology. J Cell Mol Med. 2008;12:2255-62.

29. Halle A, Hornung V, Petzold GC, Stewart CR, Monks BG, Reinheckel T, Fitzgerald KA, Latz E, Moore KJ, Golenbock DT. The NALP3 inflammasome is involved in the innate immune response to amyloid-beta. Nat Immunol. 2008;9:857-65

30. Tseng WA, Thein T, Kinnunen K, Lashkari K, Gregory MS, D'Amore PA, Ksander BR. NLRP3 inflammasome activation in retinal pigment epithelial cells by lysosomal destabilization: implications for age-related macular degeneration. Invest Ophthalmol Vis Sci. 2013;54:110-20.

31. Anderson OA, Finkelstein A, Shima DT. A2E induces IL-1ss production in retinal pigment epithelial cells via the NLRP3 inflammasome. PLoS One. 2013;8:e67263.

32. Kerur N, Hirano Y, Tarallo V, Fowler BJ, Bastos-Carvalho A, Yasuma T, Yasuma R, Kim Y, Hinton DR, Kirschning CJ, et al. TLR-independent and P2X7-dependent signaling mediate Alu RNA-induced NLRP3 inflammasome activation in geographic atrophy. Invest Ophthalmol Vis Sci. 2013;54:7395-401.

33. Kauppinen A, Niskanen H, Suuronen T, Kinnunen K, Salminen A, Kaarniranta K. Oxidative stress activates NLRP3 inflammasomes in ARPE-19 cells_-implications for age-related macular degeneration (AMD). Immunol Lett. 2012;147:29-33.

34. Doyle SL, Campbell M, Ozaki E, Salomon RG, Mori A, Kenna PF, Farrar GJ, Kiang AS, Humphries MM, Lavelle EC, et al. NLRP3 has a protective role in age-related macular degeneration through the induction of IL-18 by drusen components. Nat Med. 2012;18:791-8.

35. Doyle SL, Ozaki E, Brennan K, Humphries MM, Mulfaul K, Keaney J, Kenna PF, Maminishkis A, Kiang AS, Saunders SP, et al. IL-18 attenuates experimental choroidal neovascularization as a potential therapy for wet age-related macular degeneration. Sci Transl Med. 2014;6:230ra244.

36. Doyle SL, Lopez FJ, Celkova L, Brennan K, Mulfaul K, Ozaki E, Kenna PF, Kurali E, Hudson N, Doggett T, et al. IL-18 immunotherapy for neovascular AMD: tolerability and efficacy in nonhuman primates. Invest Ophthalmol Vis Sci. 2015:56:5424-30

37. Hirano Y, Yasuma T, Mizutani T, Fowler BJ, Tarallo V, Yasuma R, Kim Y, Bastos-Carvalho A, Kerur N, Gelfand BD, et al. IL-18 is not therapeutic for neovascular age-related macular degeneration. Nat Med. 2014;20:1372-5.

38. Liu X, Zhang Z, Ruan J, Pan Y, Magupalli VG, Wu H, Lieberman J. Inflammasome-activated gasdermin D causes pyroptosis by forming membrane pores. Nature. 2016:535:153-8.

39. Sborgi L, Ruhl S, Mulvihill E, Pipercevic J, Heilig R, Stahlberg H, Farady CJ, Muller DJ, Broz P, Hiller S. GSDMD membrane pore formation constitutes the mechanism of pyroptotic cell death. EMBO J. 2016;35:1766-78.

40. Sagulenko V, Thygesen SJ, Sester DP, Idris A, Cridland JA, Vajjhala PR, Roberts TL, Schroder K, Vince JE, Hill JM, et al. AIM2 and NLRP3 inflammasomes activate both apoptotic and pyroptotic death pathways via ASC. Cell Death Differ. 2013;20:1149-60.

41. Woodell A, Coughlin B, Kunchithapautham K, Casey S, Williamson T, Ferrell WD, Atkinson C, Jones BW, Rohrer B. Alternative complement pathway deficiency ameliorates chronic smoke-induced functional and morphological ocular injury. PLoS One. 2013;8:e67894.

42. Newman AM, Gallo NB, Hancox LS, Miller NJ, Radeke CM, Maloney MA, Cooper JB, Hageman GS, Anderson DH, Johnson LV, Radeke MJ. Systems-level analysis of age-related macular degeneration reveals global biomarkers and phenotype-specific functional networks. Genome Med. 2012;4:16.

43. Ardeljan D, Chan CC. Aging is not a disease: distinguishing age-related macular degeneration from aging. Prog Retin Eye Res. 2013;37:68-89.
44. McLeod DS, Bhutto I, Edwards MM, Silver RE, Seddon JM, Lutty GA. Distribution and quantification of choroidal macrophages in human eyes with age-related macular degeneration. Invest Ophthalmol Vis Sci. 2016;57: 5843-55.

45. Bressler SB, Bressler, N.M.: Age-related macular degeneration: nonneovascular early AMD, intermediate AMD, and geographic atrophy. In Retina (Ryan SJ ed., vol. 2, 5th edition. London: Elsevier; 2014. p. 1150-82.

\section{Submit your next manuscript to BioMed Central and we will help you at every step:}

- We accept pre-submission inquiries

- Our selector tool helps you to find the most relevant journal

- We provide round the clock customer support

- Convenient online submission

- Thorough peer review

- Inclusion in PubMed and all major indexing services

- Maximum visibility for your research

Submit your manuscript at www.biomedcentral.com/submit 